\title{
Foco activo de Malaria, Resguardo Indígena Caño Mochuelo, Casanare, Colombia 2020 - 2021.
}

\section{Active focus of Malaria, Caño Mochuelo Indigenous Reserve, Casanare, Colombia 2020-2021.}

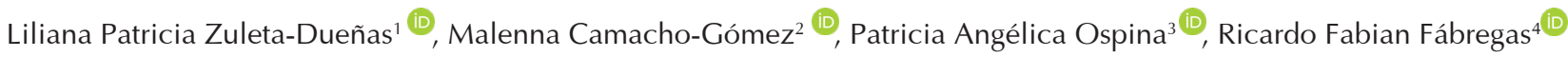

\section{RESUMEN}

Casanare en la Orinoquía colombiana, presenta bajo reporte de casos de malaria. Entre la semana epidemiológica (SE) 28 a la SE-49 de 2020 se reportaron 77 casos. $61 \%$ hombres, $9 \%$ migrantes, $2.6 \%$ malaria complicada, letalidad: $1.3 \%$ (1/77); mortalidad: 0.06/100.000 habitantes. En la SE-3 a la 13 de 2021 se notificaron 51 casos con la misma procedencia. Objetivo. Caracterizar la situación eco-epidemiológica de un foco de malaria. Materiales y métodos. Estudio descriptivo: análisis epidemiológico (Índice Parasitario Anual-IPA, tasa de mortalidad y letalidad), verificación de atención integral del paciente y estudio entomológico. Resultados. En 2021 los casos observados superaron los esperados (51 vs 7), $(p=0.000) .51 \%$ mujeres. $32 \%$ primera infancia y adolescencia, 25\% jóvenes, 39\% adultos, $4 \%$ adulto mayor, sin mortalidad, colombianos, de nueve comunidades indígenas. EI IPA en 2020 y 2021 fue 0.18 y 0.12 . La verificación diag- nostica de gota gruesa sugirió reentrenamiento del personal operativo. Anopheles braziliensis, A. darlingi, A. marajoara y A. oswaldoi se identificaron. Mayor actividad de picadura de A. darlingi: entre las 21:00h-22:00h. Entorno de mayor captura: intra-domicilio. Viviendas con intervención química: 340. Toldillos impregnados con insecticida entregados: 500. Conclusión. Se confirma la existencia de un foco receptivo en estrato 4 . Se debe priorizar el evento con acciones de vigilancia, prevención y control.

Palabras claves: Malaria, Indígena, migrante, región Orinoquía.
Citación (Vancouver): Foco activo de Malaria, Resguardo Indígena Caño Mochuelo, Casanare, Colombia 2020 - 2021. Rev Avances en Salud; 2021. (5)1 :28-29 . doi: 10.21897/25394622.2531

\footnotetext{
${ }^{1}$ Bacterióloga, Esp. epidemiología M, Sc, Salud pública, Coordinadora enfermedades trasmitidas por vectores, Secretaría de salud de Casanare.

${ }^{2}$ Bióloga, M.Sc. Gestión Ambiental, Profesional de apoyo Laboratorio de Salud pública-Entomología Medica, Secretaría de Salud de Casanare

${ }^{3}$ Enfermera, Ssp. Epidemiología, profesional de apoyo programa ETV Secretaría de Salud de Casanare ${ }^{4}$ Biólogo, profesional de apoyo programa ETV Secretaría de Salud de Casanare salud.etv@casanare.gov.co
} 


\begin{abstract}
Casanare in Orinoquia region of Colombia, have few report of cases of malaria. From epidemiological week 28 to 49 of 2020, 77 cases were reported. $61 \%$ men, $9 \%$ migrants. $2.6 \%$ severe malaria, lethality: $1.3 \%$ (1/77), mortality: $0.06 / 100,000$ inhabitants. In week 3 to 13 of 2021, 51 cases were reported from the same area. Objective. To characterize the eco-epidemiological situation of a malaria focus. Materials and methods. Descriptive study: epidemiological analysis (annual parasite index-API, mortality rate, lethality), verification to access to health care and entomological study. Results. In 2021, the observed cases exceeded those expected (51 vs 7), ( $p=0.000) .51 \%$ women. $32 \%$ from childhood to adolescence, $25 \%$ youth, $39 \%$ adults, $4 \%$ seniors, colombians. From nine indigenous communities. API in 2020 and 2021 was 0.18 and 0.12 . In the diagnostic verification of the thick blood film, it was suggested to retrain the operating personnel. Anopheles braziliensis, A. darlingi, A. marajoara and $A$. oswaldoi were identified. The higher peak biting activity of $A$. darlingi was between 21:00 to 22:00. The most mosquitoes were captured in the indoor. 340 homes fumigated. 500 insecticide treated net were given. Conclusion. Receptive focus stratum 4 is confirmed. It is required to prioritize the malaria with surveillance, prevention and control actions.
\end{abstract}

Key words: malaria, indigenous, migrant, Orinoquia region.

\section{REFERENCIAS}

1. OMS. Manual de estratificación según el riesgo de malaria y eliminación de focos de transmisión [Internet]. OMS, editor. 2019. 1-62 p. Disponible en: https://www3.paho. org/hq/index.php?option=com docman\& view=download\&slug=malaria-technicaladvisory-group-session-8-2019-onlyin-spanish\&Itemid $=270$ \&lang $=e n$

2. Ministerio de Salud y la Protección Social. Lineamiento para la gestión y operación del programa de enfermedades transmitidas por vectores y zoonosis y otras consideraciones para la ejecución de sus transferencias nacionales de funcionamiento [Internet]. Subdirección de enfermedades transmisibles grupo endemoepidémicas, editor. 2020. Disponible en: https://www.minsalud.gov. $\mathrm{co/sites/rid/Lists/BibliotecaDigital/RIDE/}$ VS/PP/lineamientos-gestion-programatransferencias-etvzoonosis-2020.pdf 